\title{
Left ventricular heart assist device using $M C$ and ECG feedback
}

\begin{abstract}
This paper aims to design and implement left ventricular heart assist device (LVAD) system. The main part of the system is a centrifugal pump that pushes blood from the left ventricle to aorta. It is controlled by Arduino Microcontroller (MC). LVAD should be placed between left ventricle and aorta of the heart using a percutaneous lead which is connected to the system controller. This system is considered as closed loop system where it is synchronized with the patient's electrocardiogram (ECG), which provides information about the patient's physiological conditions. A Brushless DC Motor is used to meet the required speed and blood flow rate according to ECG feedback signals. A prototype is designed, built and tested. The testing data are confirmed the results of the mathematical analysis. The system is constructed and programmed to operate automatically in safe mode in case of suddenly occurring failure. All data are monitored and displayed on LCD screen. The system is programmed using C language and fed by using two DC batteries, which placed on either side of the patient in specific holder
\end{abstract}

Keywords: LVAD system, centrifugal pump, ECG, heart rate
Volume 3 Issue 3 - 2017

\author{
Ramzi Qawasma,Abdel-Karim Daud \\ Electrical Engineering Department, Palestine Polytechnic \\ University, West Bank
}

\author{
Correspondence: Abdel-Karim Daud, Electrical Engineering \\ Department, Faculty of Engineering, Palestine Polytechnic \\ University, Palestine,Wet Bank, Tel +97022233050, \\ Email daud@ppu.edu
}

Received: October 04, 2017 | Published: October 20, 2017

\section{Introduction}

The human heart pumps blood throughout the body via the circulatory system, supplying oxygen and nutrients to the tissues which helps bodies function and removing carbon dioxide and other waste products. ${ }^{1}$ Cardiac failure happens when the heart cannot pump enough blood and oxygen to support other organs. ${ }^{2}$ In USA about 5.7 million adult patients are diagnosed with heart failure and one in 9 deaths in 2009 included heart failure as contributing cause. ${ }^{3}$ The European Society of Cardiology represents countries with a population of more than 900 million, and in their last guidelines they reported that there are at least 26 million patients suffering this disease in those 51 countries. ${ }^{4}$ Heart failure is a serious condition, but it does not mean that the heart has stopped beating. At present time, cardiac transplantation remains the gold standard of cardiac replacement therapy. However, the supply of donor hearts is limited and therefore is not an option for many patients because of age and other conditions. Alternative forms of cardiac replacement therapy include cell therapy, xenotransplantation, ventricle assist devices implantation and total artificial heart. Left ventricle assist device is more and more reliable, efficient and its size is becoming smaller with technology development.

LVAD is a mechanical device that circulates blood through the body when the heart is too weak to pump a sufficient amount of blood on its own. It is designed to take over the pumping function of the patient's left ventricle. It is placed just below the diaphragm in the abdomen and attached to the left ventricle, and the aorta, which is considered as the main artery that carries oxygenated blood from the left ventricle to the entire body. ${ }^{5}$ The appropriate set point for the pump rotational speed depends on mechanical properties of the LVAD, which vary dynamically with time. The slower speed of the pump is determined by the requirement to maintain adequate blood perfusion and to avoid regurgitate flow and unsatisfactory blood flow dynamics. The highest speed of the pump is limited by induction of suction in the left ventricle, which occurs when the LVAD attempts to pump more blood from the ventricle than is available. Suction can be deleterious to the myocardium, blood, and lungs. ${ }^{6}$

Three different generation groups of LVAD are available. These heterogeneous groups consisting of devices developed in different periods, employing different drive mechanisms, inspired by different principles. First LVAD generation is implanted, pulsatile, electric driven, bulky. Pusher plate displacement pumps connected to a wearable control and battery unit by a driveline piercing the skin. The main drawbacks of this generation were anticoagulation necessity and a relatively high incidence of thromboembolic events. ${ }^{7}$ Second generation is mainly named as "axial flow pump". This pump uses electrical energy to rotate an axle on which a turbine or propeller system is mounted to propose liquids forwardly. A very high rotation rate makes it possible to pump large amount of blood in accordance with the body needs. This system consists of much smaller pump with fewer moving parts and less blood contacting surface. The heat is generated by the high rotational speeds causing hemolysis that may be lead to damage the blood cells and thrombi may be occur also. ${ }^{7}$ Third generation is mainly implantable centrifugal pumps developed for long-term use. Many devices in this group are newly developed or in development stage. These pumps use centrifugal energy to propulse blood but they somehow differ from each other in many aspects such as their implantation, characteristics, dimensions, interrelation between the moving parts working principles and device-blood interface. ${ }^{7}$

This paper represents Left Ventricular Assist Device system as shown in Figure 1. It is designed to support systemic circulation of the blood when the natural heart is unable to maintain adequate perfusion with conventional therapy. To provide the system with supporting capabilities, LVAD system takes blood from the natural heart and then pumps blood to the arterial system in a pulsatile manner at normal arterial pressures. To achieve this, it is necessary to design and implement this system based on Arduino Microcontroller and using special pump. An external, wearable device is designed to hold the pump, external drivelines, controller, LCD and system 
batteries. Figure 2 shows the LVAD block diagram, where the ECG system provides the Aurduino Microcontroller with the ECG signal through a conditional circuit. This signal is analyzed to determine the R-wave. After the R-wave detection, the electrical valve, that is located between left atrium and left ventricle, will open to permit the blood to pass to the pump, which pushes the blood to the Aorta. Then the blood circulates through the body and return back to the left atrium and so on. In this manner the blood circulation is accomplished using the left ventricular heart assist device system. The described LVAD is designed, implemented and tested in the biomedical engineering laboratory at Palestine Polytechnic University.

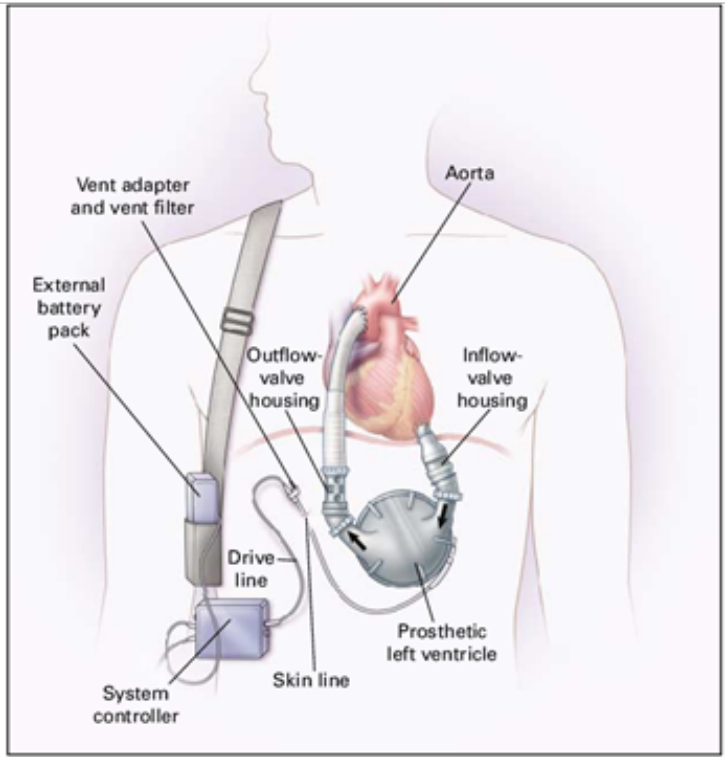

Figure I LVAD description. ${ }^{8}$

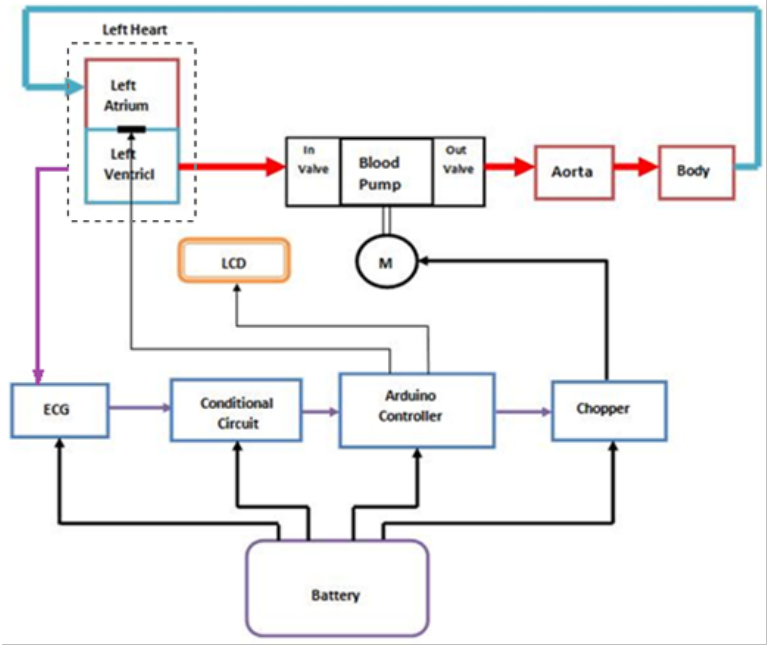

Figure 2 LVAD functional block diagram.

\section{System analysis}

The designed LVAD is consisted from four major parts: ECG recording system, controlling system, blood pumping system and emergency system. As shown previously in Figure 2, these systems are worked synchronously with the other chambers of the heart. If any changes in the heart beat occur during the operation, the LVAD will respond quickly. The general flowchart that describes the flow of information between system components is shown in Figure 3.The recorded ECG signal is directed to the conditional circuit to be analyzed in term of R-wave detection. The detection of R-wave means that the heart is still pumps but can't provide the body with a sufficient amount of blood. It used also to determine the required flow rate according to the body needs. If $\mathrm{R}$-wave is not existed in the ECG waveform the emergency system will be activated and the pump will work in a constant average rate 75 BPM to ensure enough blood supply to the body for survival. In emergency conditions sound and light alarms will be fire to warn the patient.

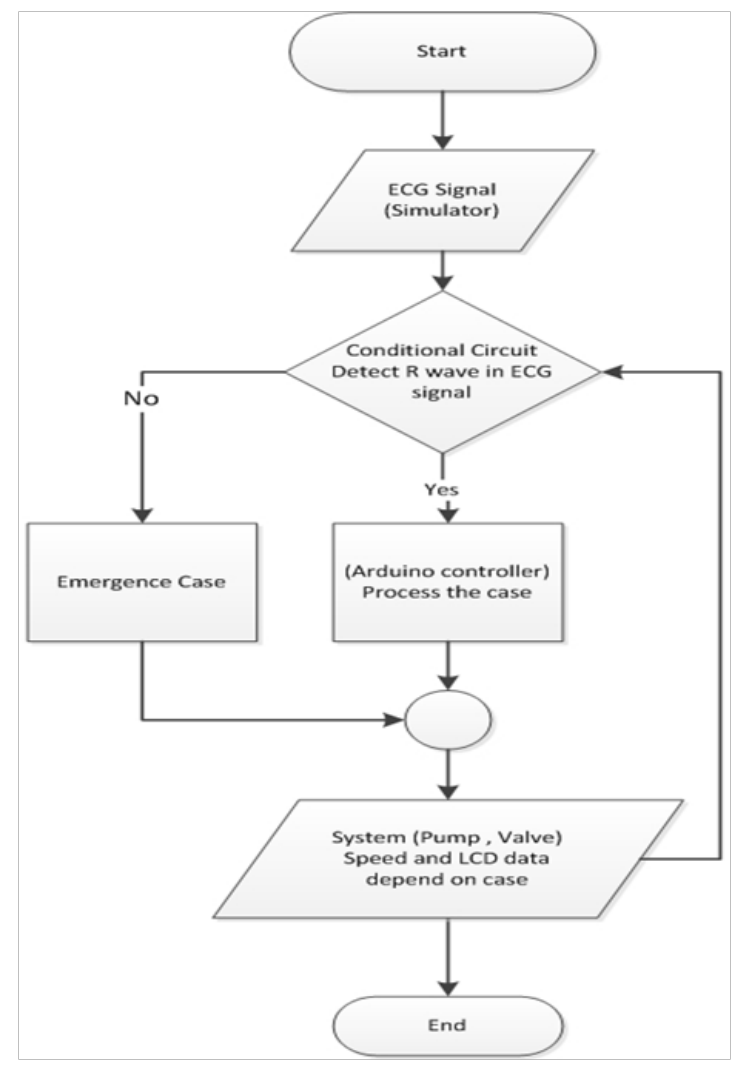

Figure 3 LVAD flowchart.

In all cases: normal rate, tachycardia, bradycardia or any other abnormalities the controller determines the speed of the motor and the flow rate. All patient data and battery status are displayed on the LCD.

\section{ECG system}

The ECG signal is recorded using second Einthoven lead (Lead II). ${ }^{9}$ The P-wave represents the atrial depolarization, while the QRScomplex represents ventricular depolarization. In the other side the ST-T-U complex (ST-segment, T- and U-waves) represents ventricular repolarization (Figure 4). Atrial repolarization is usually too low in amplitude to be detected, but it may become apparent in some pathological conditions such as acute pericarditis or atrial infarction. The R-wave amplitude of the QRS-complex, which occurs during the ventricular depolarization is relatively high, therefore it can be easily detected by the microcontroller and then the time between two successive $\mathrm{R}$-waves is measured to determine the conditions in which the heart works. The R-wave detection is very important since it is used for counting the heart rate, which determines the bodily demand from the oxygenated blood. The speed of the motor (Figure 2) depends on the heart rate (HR). While the time between each two successive R-waves is measured, the number of beats per minute is 
calculated. ${ }^{9}$ Three conditions are used for controlling the speed of the pump depending on the real time calculated heart rate from the recorded ECG signal. These conditions are tachycardia (HR $>100$ $\mathrm{BPM})$, normal heart rate $(60-100 \mathrm{BPM})$ and bradycardia $(\mathrm{HR}<60$ BPM).

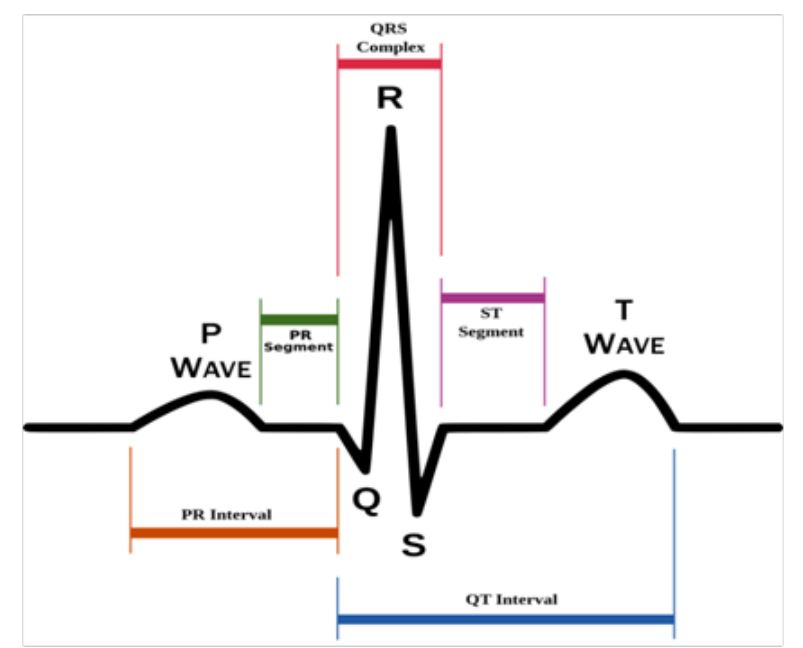

Figure 4 Basic ECG waveforms and intervals. ${ }^{9}$

Suppose that the average HR is 75 BPM, then the total time of the full ECG cycle $\left(\mathrm{T}_{\mathrm{ECG}}\right)$ is $60 \mathrm{~s} / 75 \mathrm{BPM}=800 \mathrm{~ms}$. As known the ventricles contract after $300 \mathrm{~ms}$ from the beginning of the cardiac cycle (Ventricles Contraction Time $-\mathrm{t}_{\mathrm{vC}}$ ), so a constant coefficient (CC) can be determined as $\mathrm{T}_{\mathrm{ECG}} / \mathrm{t}_{\mathrm{VC}}$ to have $\mathrm{CC}=2.667 . .^{10}$ It is clear that the ventricles contraction time changes according to the change in HR. Table 1 shows the change in $\mathrm{T}_{\mathrm{ECG}}$ and $\mathrm{t}_{\mathrm{VC}}$ for different heart rhythm.

Table I Important parameters for different heart rhythm

\begin{tabular}{lllll}
\hline No. & Rhythm & HR, BPM & $\mathbf{T}_{\mathrm{ECG}}, \mathbf{m s}$ & $\mathbf{t}_{\mathrm{vc}}, \mathbf{m s}$ \\
\hline $\mathrm{I}$ & Tachycardia & $>100$ & $<600$ & $<225$ \\
2 & Normal HR & $60-100$ & $600-1000$ & $225-375$ \\
3 & Bradycardia & $<60$ & $>1000$ & $>375$ \\
\hline
\end{tabular}

\section{Controlling system}

Many investigators have worked on control techniques for various types of LVADs. ${ }^{11-13}$ In this study the DC motor control system is designed and performed using a chopper circuit, which is used for controlling the speed of the DC motor. The programming in microcontroller analyzes the number of beats per minute, then it provides the chopper circuit with the required number of pulses to control the motor speed that is necessary to provide the body with a specific volume of the blood by PWM method. ${ }^{14}$ What distinguishes the ECG feedback is more accurate and efficient in pump and all system. Therefore, the responsibility of the system becomes so fast according to changing in heart rate. In cardiovascular physiology, stroke volume (SV) is the volume of blood pumped from the left ventricle per beat. Stroke volume is an important determinant of cardiac output (CO), which is the product of stroke volume and heart rate. The term stroke volume can apply to each of the two ventricles of the heart, although it usually refers to the left ventricle. The stroke volumes for each ventricle are generally equal, both being approximately $70 \mathrm{~mL}$ in a healthy $70-\mathrm{kg}$ man. ${ }^{15,16}$ $\{\backslash$ displaystyle $\mathrm{SVI}=\{\mathrm{SV} \backslash$ over $\mathrm{BSA}\}=\{(\mathrm{CO} / \mathrm{HR}) \backslash$ over $\mathrm{BSA}\}=\{\mathrm{CO}$ lover $\{\mathrm{HR} \backslash$ times BSA $\}\}$ Because it pumps out whatever blood comes back into it from the venous system, the quantity of blood returning to the heart effectively determines the quantity of blood the heart pumps out - its cardiac output, CO or Q. Cardiac output is classically defined alongside stroke volume (SV) and the heart rate (HR) as:

$$
\mathrm{CO}_{[\mathrm{L} / \mathrm{min}]}=\mathrm{SV}_{[\mathrm{L} / \text { beat }]} \times \mathrm{HR}_{\text {[beats/min }]}
$$

The number of beat per minute HR of the heart is given by:

$$
\mathrm{HR}=\frac{60}{\mathrm{~T}_{\mathrm{ECG}}}
$$

With the motor voltage $\mathrm{V}_{\mathrm{o}}$ according to

$$
\mathrm{V}_{\mathrm{o}}=\frac{\mathrm{HR} \times 12}{120}
$$

And the total time period $\mathrm{T}$

$$
\mathrm{T}=\frac{1}{\mathrm{f}}
$$

The on time $t_{\text {on }}$ can be described as

$$
\mathrm{t}_{\text {on }}=\frac{\mathrm{V}_{\mathrm{o}} \times \mathrm{T}}{\mathrm{V}_{\mathrm{s}}}
$$

Where $\mathrm{f}$ is the chopper frequency and $\mathrm{V}_{\mathrm{s}}$ is the supply voltage.

According to frequency modulation control, the duty cycle (D) is obtained as:

$$
\mathrm{D}=\mathrm{t}_{\text {on }} / \mathrm{T}
$$

Where the frequency remains constant and on time $\left(\mathrm{t}_{\text {on }}\right)$ is varied.

The pump and motor speed can be calculated as follows [6] :

$$
\begin{aligned}
& \mathrm{Q}=\mathrm{A} \cdot \mathrm{v} \\
& \mathrm{A}=\pi \cdot \mathrm{r}^{2} \\
& \mathrm{n}=\frac{\mathrm{v}}{\mathrm{R}}
\end{aligned}
$$

Where: $\mathrm{Q}=\mathrm{CO}$ flow rate in $\mathrm{L} / \mathrm{min}$.

$\mathrm{A}=$ cross section area to out flow tube of pump in $\mathrm{m}^{2}$

$\mathrm{v}=$ velocity of blood in $\mathrm{m} / \mathrm{min}$

$\mathrm{r}=$ radius of out tube in $\mathrm{m}$

$\mathrm{n}=$ speed of motor in rpm

$\mathrm{R}=$ radius of pump in $\mathrm{m}$

\section{Blood pumping system}

The central part of the LVAD is the blood pump, which can be used as a left, right, or biventricular assist device as required. The pump has a titanium alloy case containing an elastomeric blood-pumping sac. The blood sac is compressed by applying pressure from a pneumatic driver, thus ejecting blood from the sac to the drivelines. Similarly, the blood sac is expanded by applying vacuum from the driver, thus allowing the pre-load volumes to fill the sac with blood. Mechanical valves are mounted in the inflow and outflow ports of the blood pump control the direction of blood flow. The blood pump has an effective 
stroke volume of $65 \mathrm{ml}$ and, depending on various conditions, will pump approximately $6.5 \mathrm{~L} / \mathrm{min}$ at a rate of $100 \mathrm{bpm} .{ }^{16}$

\section{Emergence system}

As mentioned previously, LVAD is designed to work in three possible conditions: tachycardia, normal heart rate and bradycardia, depending on the R-wave repetition on the ECG signal. If an error occurs during the operation such as losing ECG signal of the patient or system failure, LVAD will operate at constant rate 75 BPM as predetermined in the programmed microcontroller. The 75 BPM is considered as the average value of heart rate, which can be used in emergency conditions to ensure a sufficient blood flow in the circulatory system. LCD is used to display the patient's vital signs and the status of the power supply (batteries). In case of any failure or low battery, sound and light alarms will fire to warn the patient.

\section{Prototype}

The four LVAD systems have been designed, implemented and tested in the biomedical engineering laboratory. Each component and system subcircuits are tested successfully. The complete LVAD circuit diagram in Figure 5 has been mounted on one printed circuit board. Figure 6 represents the final prototype of the system which was tested using different heart rate values as shown in Table 2. Experimental results prove the theoretical calculations, where the flow rate is directly proportional with heart rate. The highest flow rate was achieved in case of tachycardia, while the lowest in bradycardia. In emergency condition the system will work with average flow rate, which corresponds 75 BPM.

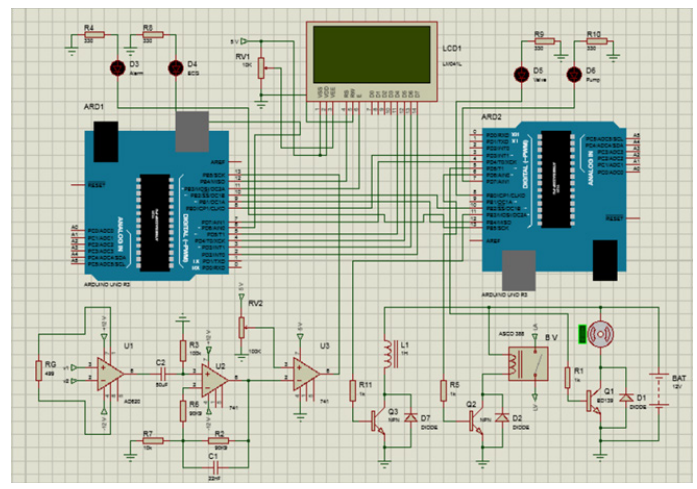

Figure 5 LVAD circuit diagram.

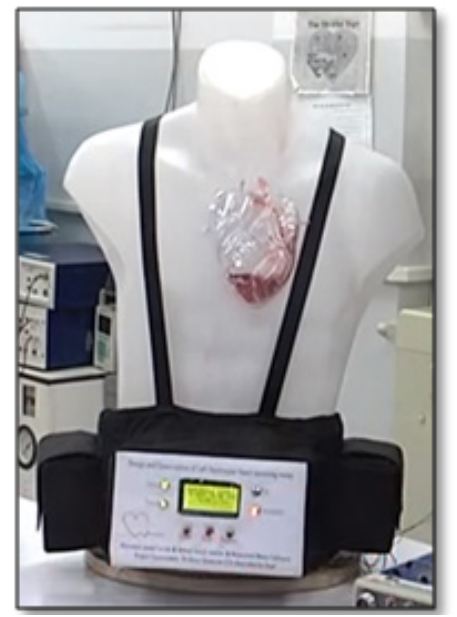

Figure 6 LVAD prototype.
Table 2 Experimental results

\begin{tabular}{llll}
\hline HR, BPM & Conditions & Pump speed, rpm & Blood flow, L/min \\
\hline 30 & Bradycardia & 1035 & 1.95 \\
60 & Normal & 2070 & 3.9 \\
90 & Normal & 3105 & 5.85 \\
120 & Tachycardia & 4140 & 7.8 \\
75 & Emergency & 2156 & 4.06 \\
\hline
\end{tabular}

\section{Conclusion}

In this paper a prototype of left ventricular heart assist device was designed, implemented and tested. All the system parts are controlled by Arduino Microcontroller. The ECG feedback provides information about the physiological status of the patient in order to adapt the speed of the DC Motor, which is considered very important parameter to determine the required blood flow rates. The system composes a very important feature, which is permitting the pump to work in a safe mode. In this mode if R-wave is not existed the emergency system will be activated and the pump will work in a constant average rate 75 BPM to ensure enough blood supply to the body cells. The experimental results show that the flow rate is directly proportional with heart rate, which is prove the theoretical calculations The highest flow rate was achieved in case of tachycardia, while the lowest in bradycardia. In emergency condition the LVAD will work with average flow rate, which corresponds 75 BPM.

\section{Acknowledgements}

This research was supported by the Deanship of Scientific Research at Palestine Polytechnic University. We are also grateful to Nabeel Jweles, Mohammed Fakhori and Mahmoud Farrah for their assistance during the implementation and testing of the system.

\section{Conflict of interest}

The author declares no conflict of interest.

\section{References}

1. Tu Jiyuan, Inthavong Kiao, Wong Kelvin, et al. Computational hemodynamics-theory, modelling and applications. Netherlands: Springer; 2015. $351 \mathrm{p}$.

2. Aurigemma GP, Gaasch WH. Clinical practice: diastolic heart failure. $N$ Engl J Med. 2004;351(11):1097-1105.

3. Mozaffarian D, Benjamin EJ, Go AS, et al. Heart disease and stroke statistics-2016 update: A report from the american heart association. Circulation. 2016;133:e38-360.

4. Ponikowski P, Anker SD, Al Habib KF, et al. Heart failure-preventing disease and death worldwide. ESC Heart Fail. 2014;1(1):4-25.

5. Shaohui Chen, James F Antaki, Marwan A Simaan, et al. Physiological control of left ventricular assist devices based on gradient of flow. American control conference; USA: IEEE; 2005. p. 3829-3834.

6. Ohuchi K, Kikugawa D, Takahashi K, et al. Control strategy for rotary blood pumps. Artificial Organs. 2001;25(5):366-370.

7. Thierry Carrela, Lars Englbergera, Michele V Martinellib, et al Continuous flow left ventricular assist devices: a valid option for heart failure patients. Swiss med wkly. 2012;142:w13701.

8. https://www.researchgate.net 
9. Rob Macleod, Brian birchler. ECG measurement and analysis; 2014.

10. Steven Deutsch, John M Tarbell, Keefe B Manning, et al. Experimental fluid mechanics of pulsatile artificial blood pumps. Annual reviews. 2006;38:65-86.

11. Gardner JF, Ignatoski M, Tasch U, et al. Aortic pressure estimation with electromechanical circulatory assist devices. J Biomech Eng. 1993;15:187-194.

12. Kitamura T. Left atrial pressure controller design for an artificial heart. Biomed Eng. 1990;37(2):164-169.
13. Shimooka T, Mitamura Y, Yuhta T. Investigation of parameter estimator and adaptive controller for assist pump by computer simulation. Artif organs. 1991;15(2):119-128.

14. Boston JR, Marwan A Simaan, James F Antalu, et al. Intelligent control design for heart assist devices april; 2009.

15. Choi S. Modeling and control of left ventricular assist system. USA: University of pittsburgh; 1998.

16. Boston JR, Simaan MA, Antaki JF, et al. Control issues in rotary heart assist devices, proceedings of the american control conference; USA: IEEE; 2000. p. 3473-3477. 